\title{
Identification of key genes, transcription factors and microRNAs involved in intracranial aneurysm
}

\author{
LIANG WEI, QI WANG, YANFEI ZHANG, CHENG YANG, HONGXIN GUAN, YIMING CHEN and ZHIYANG SUN
}

Department of Neurosurgery, East Hospital, Tongji University School of Medicine, Shanghai 200120, P.R. China

Received December 20, 2016; Accepted August 10, 2017

DOI: $10.3892 / \mathrm{mmr} .2017 .7940$

\begin{abstract}
Intracranial aneurysm (IA) is a devastating disease, the pathogenesis of which remains to be elucidated. The present study aimed to determine the molecular mechanism of IA and to identify potential therapeutic targets using bioinformatics analysis. The GSE54083 dataset, which includes data from patients with ruptured IA and superficial temporal artery controls, was downloaded from the Gene Expression Omnibus, and differentially expressed genes (DEGs) were identified in the ruptured IA samples using the limma package in R. Subsequently, the Database for Annotation, Visualization and Integrated Discovery software was used to perform function and pathway enrichment analyses and the Search Tool for the Retrieval of Interacting Genes database was used to construct the protein-protein interaction (PPI) network. Then, microRNA (miRNA) target and transcription factor (TF) target pairs were identified using the miR2Disease, MiRwalk2, ITFP and TRANSFAC databases. Finally, an integrated network of TF-target-miRNAs was constructed using Cytoscape. A total of 402 upregulated DEGs and 375 downregulated DEGs were identified from the ruptured IA samples compared with the superficial temporal artery samples. The majority of the upregulated DEGs were significantly enriched in the immune system development category, including CD40 ligand $(C D 4 O L G)$ and $C D 40$ and the downregulated DEGs, such as striatin $(S T R N)$, were enriched in neuron projection development. In addition, nitric oxide synthase 1 (NOS1), a target of miRNA-125b, and myosin heavy chain 11 (MYH11), a target of minichromosome maintenance complex component 4 (MCM4), had higher degree scores in the integrated network. These findings suggest that CD40, CD4OLG, NOS1, STRN, MCM4, MYH11 and miR-125b may be potential therapeutic targets for the treatment of IA.
\end{abstract}

Correspondence to: Dr Zhiyang Sun, Department of Neurosurgery, East Hospital, Tongji University School of Medicine, 150 Jimo Road, Pudong New Area, Shanghai 200120, P.R. China

E-mail: dfsunzy@126.com

Key words: intracranial aneurysm, differentially expressed genes, transcription factors, microRNA, integrated network

\section{Introduction}

Intracranial aneurysm (IA), also termed cerebral aneurysm, is a localized abnormal widening of a cerebral artery lumen or arterial wall due to increased intracranial pressure (1). The estimated prevalence of IA is $2.3 \%$ worldwide (2). Usually, IAs can be divided in two subtypes: Unruptured IAs (UIA) and ruptured IAs (RIA). An estimated 50-80\% of IAs will not rupture during a person's lifetime (3). As aneurysmal rupture is the main event leading to the occurrence of subarachnoid hemorrhage (4) and it often results in high disability or death rates (5), the management of UIAs for preventing RIAs is crucial (6). Genetic factors have a crucial role in regulating RIA pathogenesis (7); therefore, it is important to identify the genetic risk factors and elucidate the molecular mechanisms involved in IA.

Many genes, microRNAs (miRNAs) and transcription factors (TFs) have been associated with the progression of IA. Previous studies have revealed that the downregulation of elastin and lim domain kinase 1 may increase the risk of IA by weakening the altered arterial wall (8). In addition, the expression levels of colony stimulating factor 3 and AXL receptor tyrosine kinase are increased in patients with RIA (9). The upregulated ATP binding cassette subfamily A member 1, which is associated with intracellular lipid accumulation, may lead to IA by increasing the occurrence of inflammation in the IA wall (10). Additionally, the dysregulation of miRNA-9 is involved in IA by suppressing the proliferation and reducing the contractility of smooth muscle cells (11) and the upregulation of miRNA-92a, which negatively regulates the expression of kruppel-like factor 2 in a rabbit model, is also responsible for IA progression (12). The ETS proto-oncogene 1 TF promotes the development of IA via mediating the overexpression of monocyte chemoattractant protein-1 in IA walls (13). However, current research only partially explains the pathogenetic mechanisms of IA and further studies aimed at identifying IA-associated genes, miRNAs and TFs and elucidating their involvement in molecular mechanisms are required.

Nakaoka et al (14) have generated a gene expression profile of patients with IA and identified the differentially expressed genes (DEGs), revealing that the majority of DEGs are associated with inflammatory and immune responses. However, they did not use bioinformatics tools for an in-depth analysis of the miRNAs and TFs associated with IA. Therefore, the present study re-analyzed their expression data using a series of bioinformatics methods to identify crucial IA-associated 
genes, miRNAs and TFs that will allow the identification of the underlying mechanisms associated with IA.

\section{Materials and methods}

Affymetrix microarray data. The microarray dataset GSE54083, downloaded from the Gene Expression Omnibus (www.ncbi.nlm.nih.gov/geo), was analyzed using the GPL4133: Agilent-014850 Whole Human Genome Microarray 4x44K G4112F (Agilent Technologies, Inc., Santa Clara, CA, USA) platform. A total of 18 samples were present in this dataset, including 8 ruptured IA (RIA) and 10 superficial temporal artery (STA) samples. All 8 RIA samples were from females (age, 28-88 years) and the 10 STA samples included 8 females and 2 males (age, 34-61 years). All specimens were obtained through surgery at the Tokyo Metropolitan Fuchu Hospital (Tokyo, Japan), and all patients enrolled in the experiment provided written informed consent approved by the Ethics Committee of Tokyo Metropolitan Fuchu Hospital, Tokyo Women's University (Tokyo, Japan) (14).

Data preprocessing. Raw probe-level data was downloaded, and expression profile data preprocessing was performed using a robust multiarray average method based on the affy package (version 1.52.0; www.bioconductor.org/packages/release/bioc/html/affy.html) in R version 3.3.2 (15). Data preprocessing included background correction, filling in of missing data and quartile normalization.

Identification of DEGs and hierarchical clustering analysis. Following data preprocessing, DEGs between RIA and STA samples were analyzed using Bayes methods based using the limma package version 3.30.3 (www.bioconductor.org/packages/release/bioc/html/limma.html) in R (16), and raw P-values were revised using the Benjamini and Hochberg method (17). The cut-off criteria for defining DEGs were adjusted $\mathrm{P}<0.05$ and $\mid \log _{2}$ fold change $(F C) \mid>2$. Clustering analysis was then conducted using the pheatmap package in $\mathrm{R}$ (cran.r-project. org/web/packages/pheatmap/index.html).

Functional and pathway enrichment analyses of DEGs. The Database for Annotation, Visualization and Integrated Discovery version 6.8 (DAVID; david-d.ncifcrf.gov) provides functional classification and annotation analyses of associated genes (18). In the present study, the DAVID database was used to perform Gene Ontology (GO) and Kyoto Encyclopedia of Genes and Genomes (KEGG) enrichment analyses of upregulated and downregulated DEGs. The significant GO terms and KEGG pathways enriched with upregulated and downregulated DEGs were selected with a criterion of $\mathrm{P}<0.05$.

Protein-protein interaction (PPI) network construction. The Search Tool for the Retrieval of Interacting Genes version 9.1 (STRING; www.string-db.org) database can annotate all functional interactions among proteins by computational prediction methods (19), and the present study used STRING to predict the interactions among proteins encoded by DEGs. The PPI pairs with a combined score $>0.4$ were identified, and the interaction pairs were visualized by constructing a PPI network using Cytoscape version 3.0. Subsequently CytoNCA

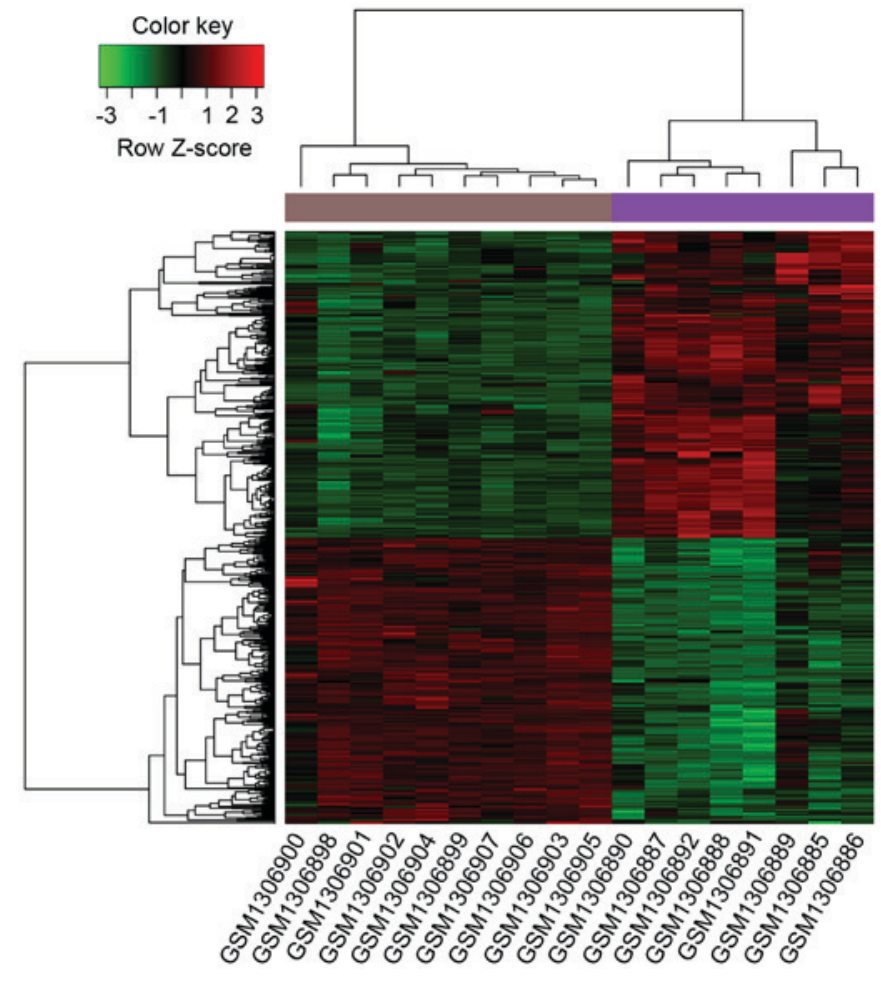

Figure 1. Hierarchical clustering map of DEGs. The horizontal axis shows the names of each sample and the right vertical axis shows the clusters of DEGs. The colored bars between the dendogram and heatmap indicate superficial temporal arteries samples in dark purple and RIA samples in light purple. Colors towards red represent high expression values and colors towards green represent low expression values. DEGs, differentially expressed genes; RIA, ruptured intracranial aneurysm.

version 2.1.6 (apps.cytoscape.org/apps/cytonca), a Cytoscape plugin, was used for centrality analysis by examining the network topology (20), and the hub nodes were identified by a high score based on the scale-free property of the network.

Integrated network construction of TF-target-miRNA. Prior to integrated network construction, the TF and miRNA targets were identified. The miR2Disease database (www.mir2disease.org) was used to search for IA-associated miRNAs (21) and the miRNA target genes were predicted from MiRwalk2, MiRDB, RNA22, miRanda, RNAhybrid and TargetScan databases. A gene was categorized as a miRNA target when identified in at least 4 out of the aforementioned 6 databases. Genes shared between predicted miRNA targets and DEGs were selected for analyses to predict whether TFs were among DEGs and miRNA targets, which were performed based on ITFP (itfp.biosino.org/itfp) and TRANSFAC (www.gene-regulation.com/pub/databases.html) databases. Subsequently, TF targets that overlapped between the predicted TF target and TF DEGs were screened. Finally, the regulation relationships between the TF-target and miRNA-target were integrated, and the TF-target-miRNA network was constructed using Cytoscape (www.cytoscape.org).

\section{Results}

$D E G$ screening and hierarchy cluster analysis. Based on the aforementioned criteria, a total of 777 DEGs were identified 
A

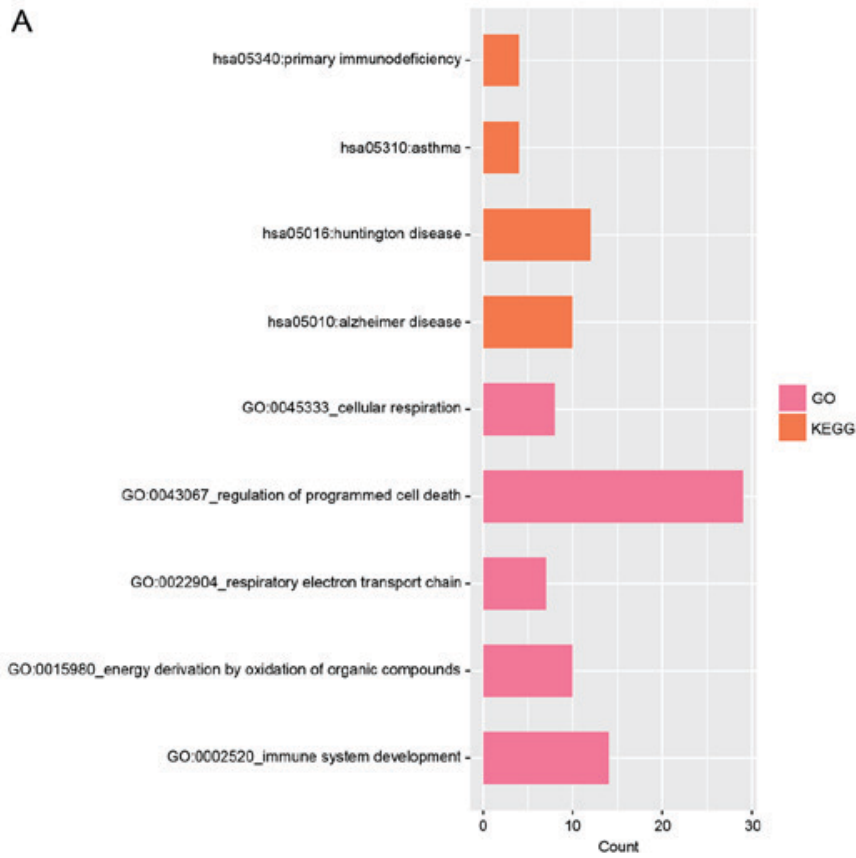

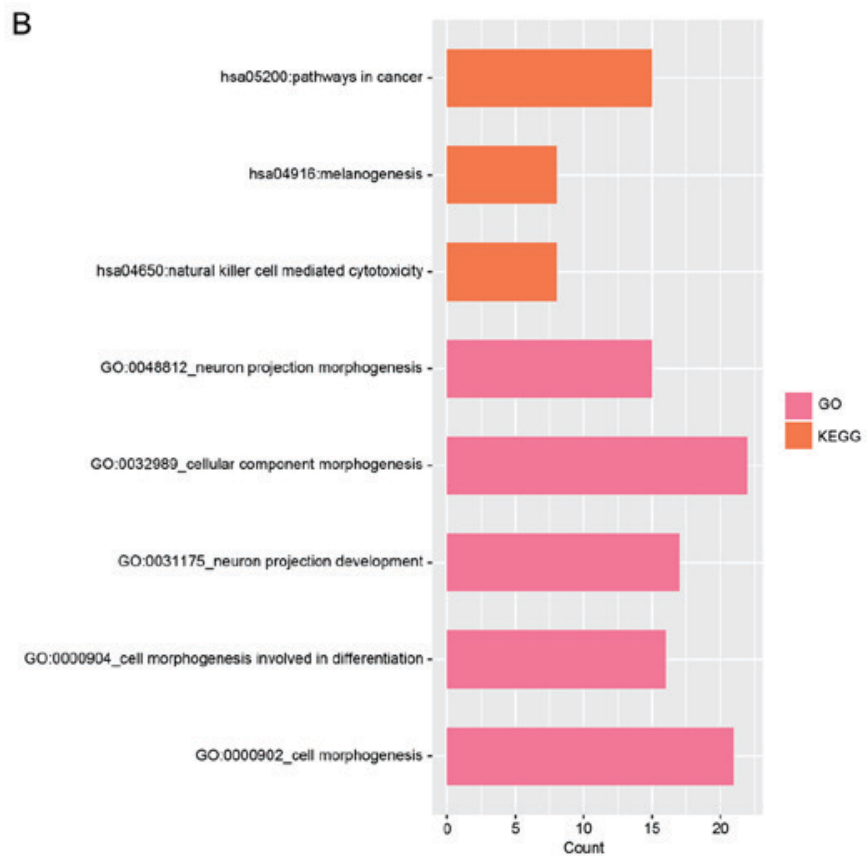

Figure 2. Function and pathway enrichment analysis. The functionally enriched GO terms and enriched pathways of (A) upregulated and (B) downregulated DEGs. GO, Gene Ontology; DEGs, differentially expressed genes.

when the RIA samples were compared with the STA samples, including 402 that were upregulated and 375 that were downregulated. In addition, the hierarchy cluster analysis indicated that DEGs could correctly distinguish between RIA and STA samples based on significantly different expression patterns, confirming that the results were reliable and could be applied to subsequent analyses (Fig. 1).

Functional and pathway enrichment analyses of DEGs. With a threshold of $\mathrm{P}<0.05$, the upregulated DEGs were enriched in $37 \mathrm{GO}$ terms and 4 KEGG pathways. The top $5 \mathrm{GO}$ terms and 4 KEGG pathways are presented in Fig. 2A and mainly involved immune system development ( $\mathrm{GO}$ terms, $\mathrm{P}=0.006$ ), regulation of programmed cell death ( $\mathrm{GO}$ terms, $\mathrm{P}=0.007)$ and primary immunodeficiency (KEGG, $\mathrm{P}=0.047$ ) categories. The downregulated DEGs were closely associated with 135 GO terms and 3 KEGG pathways, which included natural killer cell-mediated cytotoxicity (KEGG, $\mathrm{P}=0.029)$ and neuron projection development (GO terms, $\left.\mathrm{P}=1.828 \times 10^{-5}\right)$ (Fig. 2B).

PPI network analysis. PPIs encoded by DEGs were identified using the STRING database and a subset of 239 upregulated DEGs with 440 interactions was included in the upregulated PPI network (Fig. 3). For the downregulated PPI network, there were 202 DEGs and 374 interactions (Fig. 4). In addition, the top 10 upregulated nodes, including $C D 40$ and $C D 40 L G$ and downregulated nodes, such as $D R D 2$ and TGFB1 with a high degree score are presented in Table I.

Integrated network analysis. Based on the miR2Disease database, 12 IA-associated miRNAs were identified, and among these miRNAs, 9, including hsa-miR-125a, hsa-miR-125b, hsa-miR-145, hsa-miR-146a, hsa-miR-21 and hsa-miR-214 were confirmed to be regulators of the aforementioned DEGs. Additionally, a total of 18 upregulated DEGs, such as MCM4,
Table I. Top 10 upregulated and downregulated nodes of the protein-protein interaction network centrality analysis.

\section{A, Upregulated}

\begin{tabular}{lccl}
\hline Gene & Degree & Betweenness & Closeness \\
\hline KIT & 20.0 & 4938.772 & 0.07957205 \\
MAPK14 & 17.0 & 9604.479 & 0.08109029 \\
CD40 & 17.0 & 6098.7065 & 0.08153477 \\
CD79A & 14.0 & 4231.3047 & 0.0800269 \\
CD36 & 13.0 & 4260.1753 & 0.07986577 \\
CHD4 & 12.0 & 5844.3154 & 0.07989258 \\
POLR2K & 11.0 & 3625.8403 & 0.0771725 \\
SF3A2 & 11.0 & 1057.66 & 0.07345679 \\
SF3B3 & 11.0 & 1219.1407 & 0.073615834 \\
CD4OLG & 10.0 & 4938.772 & 0.07957205
\end{tabular}

B, Downregulated

\begin{tabular}{lccl}
\hline Gene & Degree & Betweenness & Closeness \\
\hline TGFB1 & 16.0 & 3138.2646 & 0.03716029 \\
EDN1 & 14.0 & 3436.2148 & 0.037284363 \\
ADCY2 & 14.0 & 3696.108 & 0.037353653 \\
SAA1 & 14.0 & 2775.5098 & 0.036921382 \\
UBL4A & 14.0 & 4874.1514 & 0.03745807 \\
CCT5 & 13.0 & 2006.9825 & 0.03694853 \\
PRKCA & 13.0 & 5347.6064 & 0.037486013 \\
DRD2 & 13.0 & 1943.1166 & 0.036914602 \\
HSPD1 & 13.0 & 2716.8848 & 0.037249815 \\
RPS20 & 12.0 & 1213.8475 & 0.03707119 \\
\hline
\end{tabular}




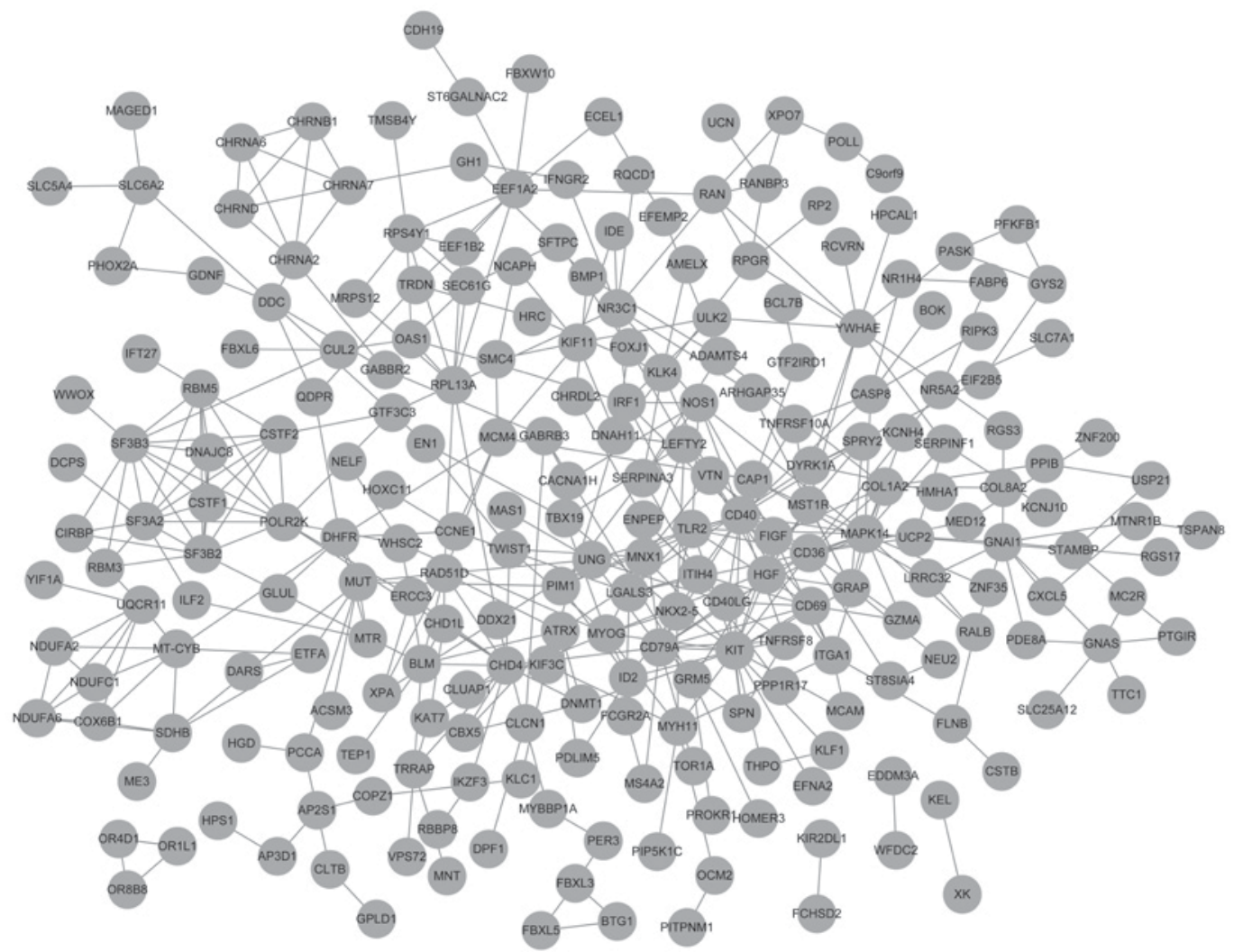

Figure 3. Protein-protein interaction network of upregulated differentially expressed genes. The nodes indicate the genes and the lines represent the corresponding interactions.

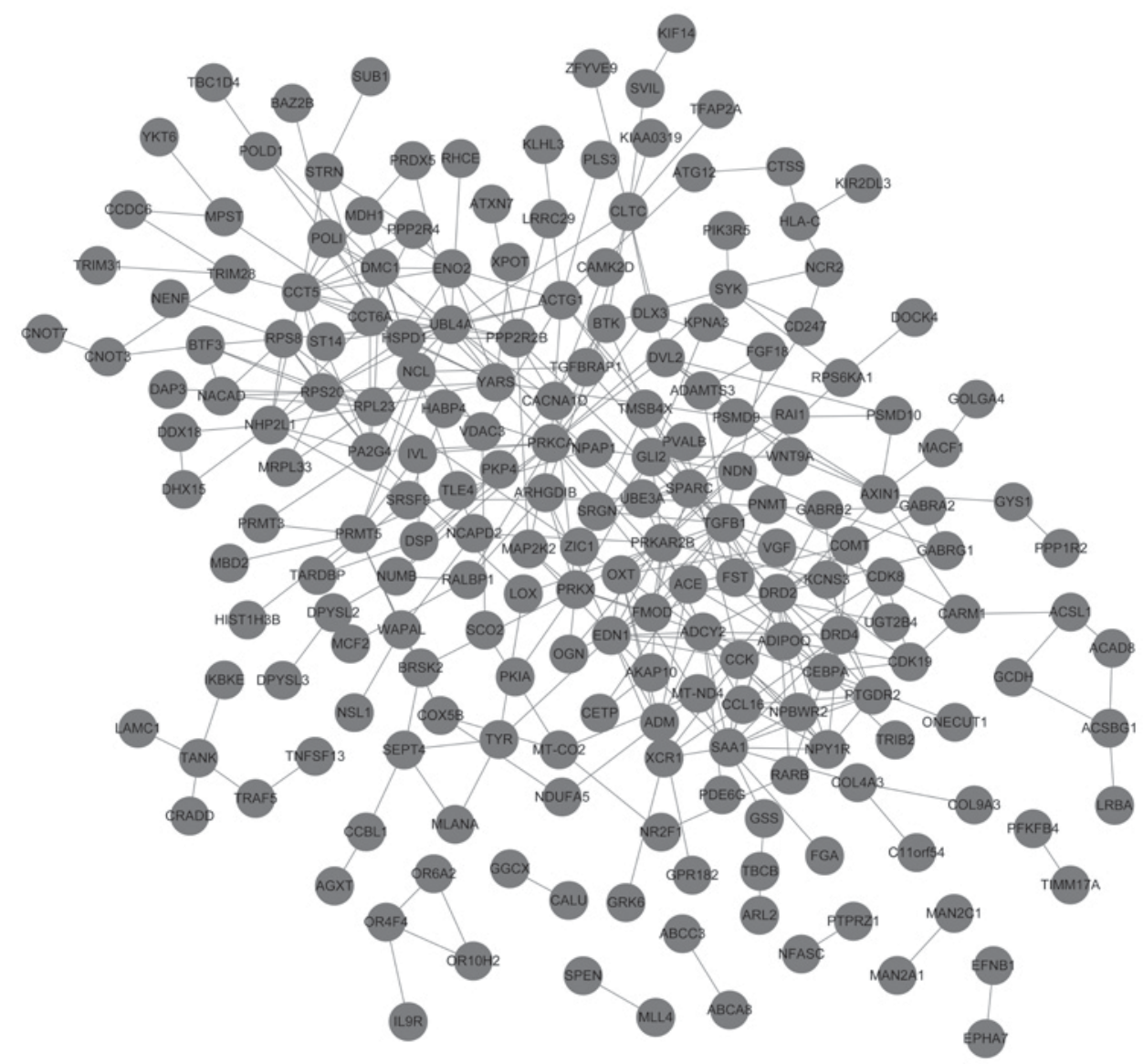

Figure 4. Protein-protein interaction network of downregulated differentially expressed genes. The nodes indicate the genes and the lines represent the corresponding interactions. 
Table II. Upregulated genes and the downregulated genes that could also be taken as transcription factors.

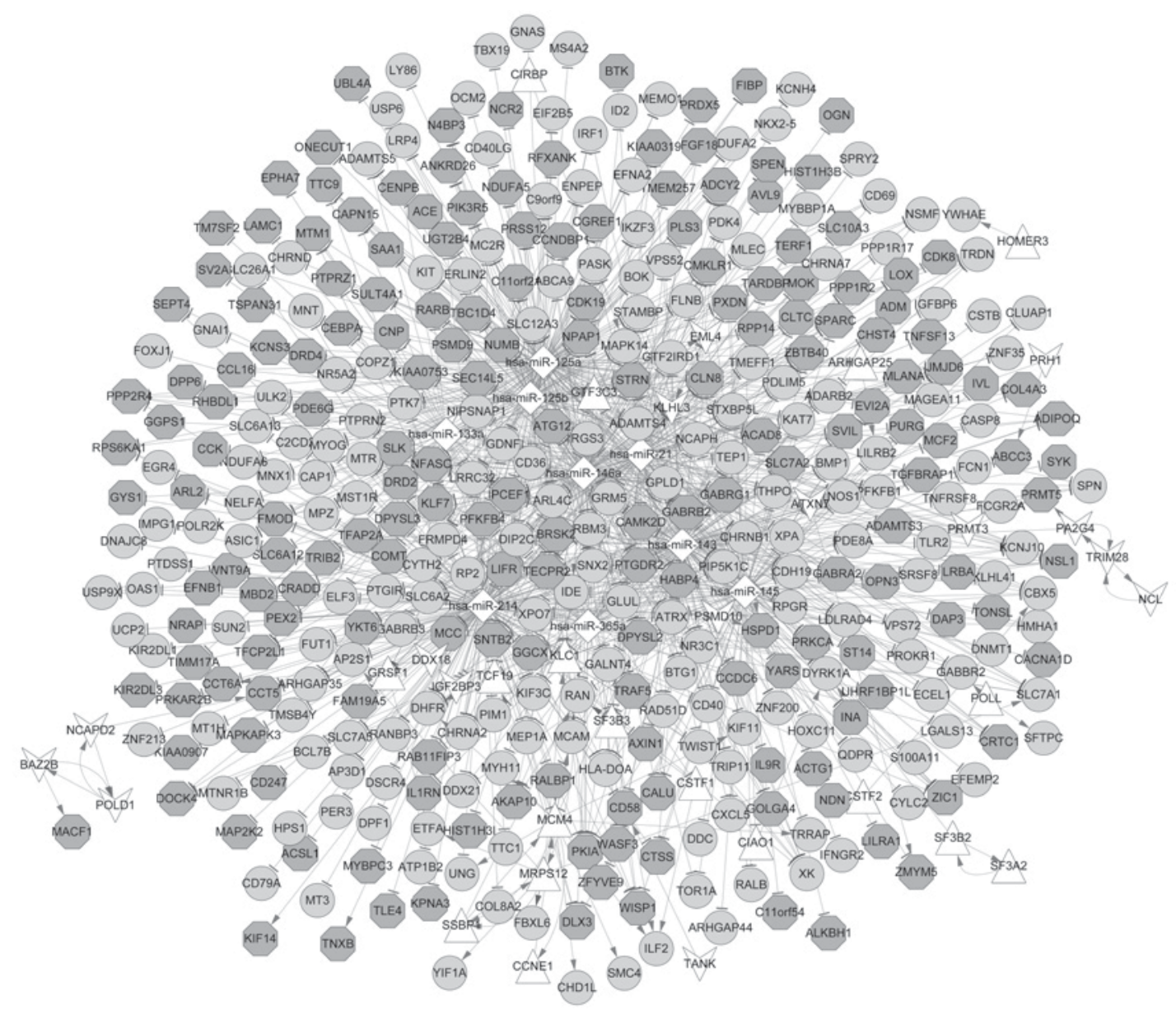

Figure 5. Integrative regulatory network of TF-target-miRNA. The circular nodes represent upregulated DEGs and the octagon nodes represent downregulated DEGs. The triangle-shaped nodes represent upregulated TFs, hexagon-shaped nodes represent downregulated TFs and diamond-shaped nodes represent miRNAs. DEGs, differentially expressed genes; TF, transcription factor; miRNA, microRNA.

MRPS12 and SF3B2 and 15 downregulated DEGs, including TRIM28 that had a regulatory function were categorized as TFs (Table II). Additionally, several DEGs with a high degree score, such as STRN and NOS1 are presented in Fig. 5.

\section{Discussion}

In the present study, 402 upregulated and 375 downregulated DEGs, were identified in the RIA samples. The majority of the DEGs were predicted to be associated with immune system development (e.g., CD4OLG and CD40) and neuron projection development (e.g., STRN) functions and in terms of pathways, such as primary immunodeficiency (e.g., CD4OLG) and natural killer cell-mediated cytotoxicity (e.g., MCM4). In addition, $C D 40$ (adjusted $\mathrm{P}=0.00001$ ) and NOS1 were hub genes in the constructed PPI networks. Additionally, DEGs, such as CD40LG (adjusted $\mathrm{P}=0.0023$ ), NOS1 (adjusted $\mathrm{P}=0.00021$ ), STRN (adjusted $\mathrm{P}=0.00011$ ) and $M Y H 11$ (adjusted $\mathrm{P}=0.0011$ ), the miRNA hsa-miR-125b and the TF MCM4 were highlighted in the TF-target-miRNA networks.

Inflammation has an important role in IA formation and rupture $(22,23)$. Prior to IA rupture, inflammatory changes in the IA walls, such as T cells and macrophage infiltration and complement activation, are major events $(24,25)$. Pyysalo et al (26) have reported that the etiology of the inflammatory alteration in patients with IA may be associated with odontogenic bacteria. Bacterial DNA was detected in 58\% IA samples from the ruptured IA sac tissue, and the overexpression of toll-like receptor 2 (TLR2) has also been reported (26), suggesting that the inflammation was bacteria-driven. Similarly, 
the findings of the present study revealed that the expression of TLR2 (adjusted $\mathrm{P}=0.00002$ ) was upregulated in IA samples. In addition, the present study revealed that the majority of the upregulated DEGs were enriched in the function of 'immune system development' (e.g., CD40LG and CD40) and the pathway of 'primary immunodeficiency' (e.g., CD4OLG). The CD40 molecule (CD40) is expressed on macrophages, and matrix metalloproteinase-2 activation in the $C D 40 L G$ pathway is crucial for abdominal aortic aneurysm (27). In addition, Ochs et al (28) have indicated that mutation of CD4OLG may lead to primary immunodeficiency, and T-cell immunodeficiency may function as a key player in the formation of IA. Therefore, this suggested that CD40 and CD4OLG contribute to the progression of IA by participating in immune system development and primary immunodeficiency. Additionally, striatin $(S T R N)$, a neuronal calmodulin-binding protein, was downregulated in neuronal dendrites and spines (29), and the present study found 17 downregulated DEGs (e.g., STRN) that were predicted to be enriched in neuron projection development. Therefore, the present study suggested that STRN may be associated with the development of IA via influencing the neuron projections development of IA cells.

Liu et al (30) have demonstrated that miR-125b, which is involved in modulating the cell proliferation of vascular smooth muscle cells, is closely associated with IA. The integrated network analysis revealed that nitric oxide synthase 1 (NOS1), a target gene of miR-125b, was predicted to be associated with IA. The overexpression of NOS1 (also termed neuronal NOS) may promote vascular nitric oxide (NO) release, and NO is responsible for macrophage-mediated vascular smooth muscle cell apoptosis $(31,32)$. Therefore, the present study proposed that regulation of NOS1 by miR-125b may contribute to the progression of IA via modulating cell proliferation and apoptosis of vascular smooth muscle cells. Additionally, the mutation of minichromosome maintenance complex component 4 (MCM4) is involved in natural killer cell deficiency in humans (33), and the natural killer cell cytotoxicity pathway is closely associated with abdominal aortic aneurysms (34). In the present study, the MCM4 TF was shown to be at a high degree in the integrated network and was predicted to regulate the expression of $M Y H 11$. Additionally, mutation of myosin heavy chain 11 (MYH11) was inversely correlated with RIA (35), which suggested that the MCM4 TF regulates $M Y H 11$ for participating in the development of IA.

In conclusion, immune system development-associated DEGs, such as CD4OLG and CD40, and regulation of cell proliferation and apoptosis of vascular smooth muscle cells by DEGs and miRNAs, such as NOS1 and miR-125b, as well as neuron projection development-associated DEGs, such as $S T R N$, may contribute to the progression of IA. Additionally, these may provide potential therapeutic targets and act as diagnostic biomarkers for IA. However, further studies are required to verify these findings.

\section{Acknowledgements}

The present study was supported by Shanghai Municipal Commission of Health and Family Planning (grant no. 201440319) and Pudong New Area Municipal Commission of Health and Family Planning (grant no. PW2014A-15).

\section{References}

1. Chalouhi N, Hoh BL and Hasan D: Review of cerebral aneurysm formation, growth, and rupture. Stroke 44: 3613-3622, 2013.

2. Blignaut Gerrit, Loggenberg Eugene, Vries D and Coert: The radiological appearance of intracranial aneurysms in adults infected with the human immunodeficiency virus (HIV). Orig Res Sa J Radiol 18: 1-4, 2014

3. Brown RD Jr: Management of cerebral aneurysms. Mayo Clinic Proceedings 79: 288-302, 2004.

4. Olafsson E,Hauser WA and Gudmundsson G: A population-based study of prognosis of ruptured cerebral aneurysm: Mortality and recurrence of subarachnoid hemorrhage. Neurology 48: 1191-1195, 1997.

5. Varble $\mathrm{N}$ and Meng $\mathrm{H}$ : Vortex imprints at the wall, but not in the bulk, distinguish ruptured from unruptured intracranial aneurysms. Presented at the DFD15 Meeting of The American Physical Society. (abstract L23.00008), 2015. http://meetings.aps. org/link/BAPS.2015.DFD.L23.8.

6. Lall RR, Eddleman CS, Bendok BR and Batjer HH: Unruptured intracranial aneurysms and the assessment of rupture risk based on anatomical and morphological factors: Sifting through the sands of data. Neurosurg Focus 26: E2, 2009.

7. Mohan D, Munteanu V, Coman T and Ciurea AV: Genetic factors involves in intracranial aneurysms-actualities. J Med Life 8: 336-341, 2015.

8. Akagawa H, Tajima A, Sakamoto Y, Krischek B, Yoneyama T, Kasuya H, Onda H, Hori T, Kubota M, Machida T, et al: A haplotype spanning two genes, ELN and LIMK1, decreases their transcripts and confers susceptibility to intracranial aneurysms. Hum Mol Genet 15: 1722-1734, 2006.

9. Xu J, Ma F, Yan W, Qiao S, Xu S, Li Y, Luo J, Zhang J and Jin J: Identification of the soluble form of tyrosine kinase receptor Axl as a potential biomarker for intracranial aneurysm rupture. BMC Neurol 15: 23, 2015.

10. Ollikainen E, Tulamo R, Lehti S, Lee-Rueckert M, Hernesniemi J, Niemelä M, Ylä-Herttuala S, Kovanen PT and Frösen J: Smooth muscle cell foam cell formation, apolipoproteins and ABCA1 in intracranial aneurysms: Implications for lipid accumulation as a promoter of aneurysm wall rupture. J Neuropathol Exp Neurol 75: 689-699, 2016.

11. Luo J, Jin H, Jiang Y, Ge H, Wang J and Li Y: Aberrant Expression of microRNA-9 contributes to development of intracranial aneurysm by suppressing proliferation and reducing contractility of smooth muscle cells. Med Sci Monit 22: 4247-4253, 2016.

12. Wu X,Zhang J,Huang Q, Yang P, Chen J and Liu J: MicroRNA-92a regulates expression of kruppel-like factor 2 in rabbit model of intracranial aneurysm. Cell Mol Biol (Noisy-le-grand) 61: 44-48, 2015.

13. Aoki T, Kataoka H, Nishimura M, Ishibashi R, Morishita R and Miyamoto S: Ets-1 promotes the progression of cerebral aneurysm by inducing the expression of MCP-1 in vascular smooth muscle cells. Gene Ther 17: 1117-1123, 2010.

14. Nakaoka H, Tajima A, Yoneyama T, Hosomichi K, Kasuya $H$, Mizutani $\mathrm{T}$ and Inoue I: Gene expression profiling reveals distinct molecular signatures associated with the rupture of intracranial aneurysm. Stroke 45: 2239-2245, 2014.

15. Irizarry RA, Hobbs B, Collin F, Beazer-Barclay YD, Antonellis KJ, Scherf U and Speed TP: Exploration, normalization, and summaries of high density oligonucleotide array probe level data. Biostatistics 4: 249-264, 2003.

16. Li C, Wei Z and Li H: Network-based empirical bayes methods for linear models with applications to genomic data. J Biopharm Stat 20: 209-222, 2010.

17. Benjamini Y and Hochberg Y: Controlling the false discovery rate: A practical and powerful approach to multiple testing. J R Statist Soc B 57: 289-300, 1995.

18. Huang da W, Sherman BT and Lempicki RA: Systematic and integrative analysis of large gene lists using DAVID bioinformatics resources. Nat Protoc 4: 44-57, 2009.

19. Franceschini A, Szklarczyk D, Frankild S, Kuhn M, Simonovic M, Roth A, Lin J, Minguez P, Bork P, von Mering C and Jensen LJ: STRING v9.1: Protein-protein interaction networks, with increased coverage and integration. Nucleic Acids Res 41 (Database issue): D808-D815, 2013.

20. Tang Y, Li M, Wang J, Pan Y and Wu FX: CytoNCA: A cytoscape plugin for centrality analysis and evaluation of protein interaction networks. Bio Systems 127: 67-72, 2015. 
21. Jiang Q, Wang Y, Hao Y, Juan L, Teng M, Zhang X, Li M, Wang G and Liu Y: miR2Disease: A manually curated database for microRNA deregulation in human disease. Nucleic Acids Res 37 (Database issue): D98-D104, 2009.

22. Chalouhi N, Ali MS, Jabbour PM, Tjoumakaris SI, Gonzalez LF, Rosenwasser RH, Koch WJ and Dumont AS: Biology of intracranial aneurysms: Role of inflammation. J Cereb Blood Flow Metab 32: 1659-1676, 2012.

23. Aoki T, Fukuda M and Narumiya S: Chronic inflammation in intracranial aneurysm formation. Inflamm Regen 33: 283-287, 2013.

24. Tulamo R, Frösen J, Junnikkala S, Paetau A, Pitkäniemi J, Kangasniemi M, Niemelä M, Jääskeläinen J, Jokitalo E, Karatas A, et al: Complement activation associates with saccular cerebral artery aneurysm wall degeneration and rupture. Neurosurgery 59: 1076-1077, 2006.

25. Kataoka K, Taneda M, Asai T, Kinoshita A, Ito M and Kuroda R Structural fragility and inflammatory response of ruptured cerebral aneurysms. A comparative study between ruptured and unruptured cerebral aneurysms. Stroke 30: 1396-1401, 1999.

26. Pyysalo MJ, Pyysalo LM, Pessi T, Karhunen PJ and Öhman JE: The connection between ruptured cerebral aneurysms and odontogenic bacteria. Öhman JE 84: 1214-1218, 2013.

27. Nagashima H, Aoka Y, Sakomura Y, Uto K, Sakuta A, Aomi S, Kurosawa H, Hagiwara N, Kawana M and Kasanuki H: Matrix metalloproteinase 2 is suppressed by trapidil, a CD40-CD40 ligand pathway inhibitor, in human abdominal aortic aneurysm wall. J Vasc Surg 39: 447-453, 2004.

28. Ochs HD, Hollenbaugh D and Aruffo A: The role of CD40L (gp39)/CD40 in T/B cell interaction and primary immunodeficiency. Semin Immunol 6: 337-341, 1994.

29. Bartoli M, Ternaux JP, Forni C, Portalier P, Salin P, Amalric M and Monneron A: Down-regulation of striatin, a neuronal calmodulin-binding protein, impairs rat locomotor activity. J Neurobiol 40: 234-243, 1999.
30. Liu D, Han L, Wu X, Yang X, Zhang Q and Jiang F: Genome-wide microRNA changes in human intracranial aneurysms. BMC Neurol 14: 188, 2014.

31. Boyle JJ, Weissberg PL and Bennett MR: Human macrophage-induced vascular smooth muscle cell apoptosis requires NO enhancement of Fas/Fas-L interactions. Arterioscler Thromb Vasc Biol 22: 1624-1630, 2002.

32. Li J, Feng W, Chen L and He J: Downregulation of SMC1A inhibits growth and increases apoptosis and chemosensitivity of colorectal cancer cells. J Int Med Res 44: 67-74, 2016.

33. Hughes CR, Guasti L, Meimaridou E, Chuang CH, Schimenti JC, King PJ, Costigan C, Clark AJ and Metherell LA: MCM4 mutation causes adrenal failure, short stature, and natural killer cell deficiency in humans. J Clin Invest 122: 814-820, 2012.

34. Hinterseher I, Schworer CM, Lillvis JH, Stahl E, Erdman R, Gatalica Z, Tromp G and Kuivaniemi H: Immunohistochemical analysis of the natural killer cell cytotoxicity pathway in human abdominal aortic aneurysms. Int J Mol Sci 16: 11196-11212, 2015.

35. Ravindra VM, Karsy M, Schmidt RH, Taussky P, Park MS and Bollo RJ: Rapid de novo aneurysm formation after clipping of a ruptured middle cerebral artery aneurysm in an infant with an MYH11 mutation. J Neurosurg Pediatr 18: 463-470, 2016.

This work is licensed under a Creative Commons Attribution-NonCommercial-NoDerivatives 4.0 International (CC BY-NC-ND 4.0) License. 